\title{
Analysis and simulation of progressive adolescent scoliosis by biomechanical growth modulation
} Ian AF Stokes

\begin{abstract}
Address: Department of Orthopaedics and Rehabilitation, University of Vermont, 434 Stafford Hall, Burlington, Vermont 05405-008, USA
\end{abstract} Email: Ian AF Stokes - Ian.Stokes@uvm.edu

from 4th International Conference on Conservative Management of Spinal Deformities Boston, MA, USA. 13-16 May 2007

Published: 12 October 2007

Scoliosis 2007, 2(Suppl I):PI2 doi:10.II86/I748-7|6I-2-SI-PI2

This abstract is available from: http://www.scoliosisjournal.com/content/2/SI/PI2

(C) 2007 Stokes; licensee BioMed Central Ltd.

\section{Objective}

Scoliosis is thought to progress during growth because angular deformity produces asymmetrical spinal loading, generating asymmetrical growth, etc. in a 'vicious cycle' [1]. The aim of this study was to test quantitatively whether calculated loading asymmetry of a spine with scoliosis, together with measured bone growth sensitivity to altered compression can explain the observed rate of progression during adolescent growth.

\section{Study design}

Estimated level-specific spinal loading asymmetry, together with the relationship expressing growth sensitivity to load were included in an analysis that was used to estimate the resulting asymmetrical vertebral growth, and its contribution to the progression of a scoliosis curvature. The initial geometry represented a lumbar scoliosis of $26^{\circ}$ Cobb, averaged and scaled from measurements of fifteen patients' radiographs. Asymmetrical loading of spine was calculated, assuming physiologically plausible muscle activation strategies. Growth sensitivity to stress was obtained from published values derived from animal studies of vertebral and tibial growth plates, expressed in a linear formulation of growth as a function of stress. Human adolescent spinal growth velocity was obtained from published values based on stereo-radiographs of a population with scoliosis.

\section{Results}

Mechanically modulated growth of the spine having an initial $26^{\circ}$ Cobb scoliosis was predicted to progress for the majority of eleven analyzed loading conditions (effort magnitude and direction). The averaged final lumbar spinal curve magnitude was $34^{\circ} \mathrm{Cobb}$ at age 16 years when the efforts producing the spinal loading were at $50 \%$ of maximum effort, and it was $42^{\circ}$ Cobb when the efforts were at $75 \%$ of maximum.

\section{Discussion}

An analysis that included analytically determined spinal load asymmetry and empirically determined growth sensitivity to load predicted that a substantial component of scoliosis progression during growth is biomechanically mediated. The rationale for conservative management of scoliosis during skeletal growth assumes a biomechanical mode of deformity progression (Hueter-Volkmann principle). The present study provides a quantitative basis for this previously qualitative hypothesis. The findings suggest that an important difference between progressive and non-progressive scoliosis might lie in the differing muscle activation strategies adopted by individuals, leading to the possibility of improved prognosis and conservative or less invasive interventions.

\section{Acknowledgements}

Supported by NIH ROI AR 44II 9 and NIH ROI AR 46543.

\section{References}

I. Stokes IAF, Windisch L: Vertebral height growth predominates over intervertebral disc height growth in adolescents with scoliosis. Spine 2006, 31:1600-1604. 